\title{
Study of Properties and Structure of Aromatic Ester Solvents
}

\author{
Santiago Aparicio, ${ }^{*}$ Rafael Alcalde, Begoña García, and José M. Leal \\ Universidad de Burgos, Departamento de Química, 09001 Burgos, Spain
}

Corresponding author: sapar@ubu.es

Supporting Information 
Table S1. Experimental Density, $\rho\left(\mathrm{g} \mathrm{cm}^{-3}\right)$, of Pure Solvents as a Function of Pressure and Temperature

\begin{tabular}{|c|c|c|c|c|c|c|c|c|c|}
\hline \multirow[b]{2}{*}{$\begin{array}{c}P \\
(\mathrm{MPa})\end{array}$} & \multicolumn{9}{|c|}{$T(\mathrm{~K})$} \\
\hline & 278.15 & 288.15 & 298.15 & 308.15 & 318.15 & 328.15 & 338.15 & 348.15 & 358.15 \\
\hline & & & & & MB & & & & \\
\hline 0.10 & 1.1015 & 1.0921 & 1.0827 & 1.0730 & 1.0633 & 1.0536 & 1.0441 & 1.0344 & 1.0249 \\
\hline 1.00 & 1.1021 & 1.0926 & 1.0832 & 1.0736 & 1.0638 & 1.0543 & 1.0448 & 1.0351 & 1.0256 \\
\hline 5.00 & 1.1044 & 1.0950 & 1.0858 & 1.0762 & 1.0667 & 1.0572 & 1.0480 & 1.0384 & 1.0291 \\
\hline 10.00 & 1.1072 & 1.0979 & 1.0890 & 1.0795 & 1.0701 & 1.0608 & 1.0517 & 1.0424 & 1.0333 \\
\hline 15.00 & 1.1099 & 1.1008 & 1.0918 & 1.0826 & 1.0734 & 1.0643 & 1.0553 & 1.0462 & 1.0373 \\
\hline 20.00 & 1.1126 & 1.1036 & 1.0948 & 1.0857 & 1.0766 & 1.0676 & 1.0588 & 1.0498 & 1.0412 \\
\hline 25.00 & 1.1152 & 1.1063 & 1.0976 & 1.0886 & 1.0797 & 1.0709 & 1.0622 & 1.0534 & 1.0449 \\
\hline 30.00 & 1.1177 & 1.1090 & 1.1004 & 1.0915 & 1.0828 & 1.0740 & 1.0655 & 1.0569 & 1.0485 \\
\hline 35.00 & 1.1202 & 1.1116 & 1.1031 & 1.0943 & 1.0857 & 1.0771 & 1.0688 & 1.0603 & 1.0520 \\
\hline 40.00 & 1.1227 & 1.1141 & 1.1057 & 1.0971 & 1.0886 & 1.0801 & 1.0719 & 1.0635 & 1.0554 \\
\hline 45.00 & 1.1251 & 1.1166 & 1.1083 & 1.0998 & 1.0914 & 1.0830 & 1.0749 & 1.0667 & 1.0587 \\
\hline 50.00 & 1.1274 & 1.1191 & 1.1109 & 1.1024 & 1.0941 & 1.0859 & 1.0779 & 1.0698 & 1.0619 \\
\hline 55.00 & 1.1297 & 1.1214 & 1.1134 & 1.1050 & 1.0968 & 1.0887 & 1.0808 & 1.0728 & 1.0651 \\
\hline \multirow[t]{2}{*}{60.00} & 1.1318 & 1.1236 & 1.1156 & 1.1074 & 1.0993 & 1.0912 & 1.0835 & 1.0755 & 1.0679 \\
\hline & & & & & EB & & & & \\
\hline 0.10 & 1.0594 & 1.0503 & 1.0409 & 1.0316 & 1.0221 & 1.0128 & 1.0034 & 0.9941 & 0.9842 \\
\hline 1.00 & 1.0599 & 1.0508 & 1.0415 & 1.0322 & 1.0228 & 1.0135 & 1.0042 & 0.9949 & 0.9850 \\
\hline 5.00 & 1.0623 & 1.0534 & 1.0442 & 1.0350 & 1.0258 & 1.0166 & 1.0075 & 0.9983 & 0.9886 \\
\hline 10.00 & 1.0652 & 1.0564 & 1.0474 & 1.0384 & 1.0293 & 1.0204 & 1.0113 & 1.0024 & 0.9930 \\
\hline 15.00 & 1.0680 & 1.0594 & 1.0505 & 1.0416 & 1.0327 & 1.0239 & 1.0151 & 1.0064 & 0.9971 \\
\hline 20.00 & 1.0708 & 1.0623 & 1.0535 & 1.0447 & 1.0360 & 1.0274 & 1.0188 & 1.0102 & 1.0011 \\
\hline 25.00 & 1.0735 & 1.0651 & 1.0565 & 1.0478 & 1.0392 & 1.0307 & 1.0222 & 1.0138 & 1.0050 \\
\hline 30.00 & 1.0761 & 1.0678 & 1.0593 & 1.0508 & 1.0423 & 1.0340 & 1.0256 & 1.0174 & 1.0087 \\
\hline 35.00 & 1.0787 & 1.0705 & 1.0621 & 1.0537 & 1.0454 & 1.0371 & 1.0289 & 1.0208 & 1.0123 \\
\hline 40.00 & 1.0812 & 1.0732 & 1.0648 & 1.0565 & 1.0483 & 1.0402 & 1.0321 & 1.0241 & 1.0158 \\
\hline 45.00 & 1.0837 & 1.0758 & 1.0675 & 1.0593 & 1.0512 & 1.0432 & 1.0353 & 1.0274 & 1.0192 \\
\hline 50.00 & 1.0861 & 1.0783 & 1.0701 & 1.0620 & 1.0540 & 1.0461 & 1.0383 & 1.0306 & 1.0225 \\
\hline 55.00 & 1.0885 & 1.0807 & 1.0726 & 1.0646 & 1.0567 & 1.0489 & 1.0412 & 1.0336 & 1.0256 \\
\hline \multirow[t]{2}{*}{60.00} & 1.0908 & 1.0831 & 1.0750 & 1.0671 & 1.0592 & 1.0516 & 1.0440 & 1.0363 & 1.0285 \\
\hline & & & & & PB & & & & \\
\hline 0.10 & 1.0339 & 1.0251 & 1.0160 & 1.0070 & 0.9982 & 0.9891 & 0.9800 & 0.9713 & 0.9621 \\
\hline 1.00 & 1.0344 & 1.0256 & 1.0166 & 1.0076 & 0.9989 & 0.9898 & 0.9808 & 0.9720 & 0.9629 \\
\hline 5.00 & 1.0368 & 1.0281 & 1.0192 & 1.0104 & 1.0017 & 0.9928 & 0.9840 & 0.9753 & 0.9664 \\
\hline 10.00 & 1.0397 & 1.0311 & 1.0223 & 1.0137 & 1.0052 & 0.9965 & 0.9878 & 0.9794 & 0.9707 \\
\hline 15.00 & 1.0425 & 1.0341 & 1.0254 & 1.0169 & 1.0086 & 1.0000 & 0.9916 & 0.9832 & 0.9748 \\
\hline 20.00 & 1.0452 & 1.0369 & 1.0284 & 1.0200 & 1.0118 & 1.0034 & 0.9951 & 0.9870 & 0.9787 \\
\hline 25.00 & 1.0479 & 1.0397 & 1.0313 & 1.0230 & 1.0150 & 1.0067 & 0.9985 & 0.9906 & 0.9824 \\
\hline 30.00 & 1.0505 & 1.0424 & 1.0341 & 1.0259 & 1.0180 & 1.0099 & 1.0019 & 0.9941 & 0.9861 \\
\hline 35.00 & 1.0530 & 1.0451 & 1.0369 & 1.0288 & 1.0210 & 1.0130 & 1.0051 & 0.9975 & 0.9896 \\
\hline 40.00 & 1.0555 & 1.0477 & 1.0396 & 1.0316 & 1.0239 & 1.0160 & 1.0083 & 1.0007 & 0.9930 \\
\hline 45.00 & 1.0580 & 1.0502 & 1.0422 & 1.0343 & 1.0267 & 1.0190 & 1.0113 & 1.0039 & 0.9963 \\
\hline 50.00 & 1.0604 & 1.0527 & 1.0448 & 1.0370 & 1.0295 & 1.0219 & 1.0143 & 1.0070 & 0.9994 \\
\hline 55.00 & 1.0627 & 1.0551 & 1.0473 & 1.0396 & 1.0322 & 1.0246 & 1.0172 & 1.0100 & 1.0025 \\
\hline 60.00 & 1.0649 & 1.0573 & 1.0498 & 1.0420 & 1.0347 & 1.0274 & 1.0200 & 1.0127 & 1.0054 \\
\hline
\end{tabular}


Table S1. Continued

\begin{tabular}{|c|c|c|c|c|c|c|c|c|c|}
\hline \multirow[b]{2}{*}{$\begin{array}{c}P \\
(\mathrm{MPa})\end{array}$} & \multicolumn{9}{|c|}{$T(\mathrm{~K})$} \\
\hline & 278.15 & 288.15 & 298.15 & 308.15 & 318.15 & 328.15 & 338.15 & 348.15 & 358.15 \\
\hline & & & & & BB & & & & \\
\hline 0.10 & 1.0176 & 1.0091 & 1.0005 & 0.9920 & 0.9834 & 0.9747 & 0.9662 & 0.9579 & 0.9495 \\
\hline 1.00 & 1.0182 & 1.0097 & 1.0010 & 0.9926 & 0.9840 & 0.9754 & 0.9669 & 0.9586 & 0.9502 \\
\hline 5.00 & 1.0205 & 1.0121 & 1.0035 & 0.9953 & 0.9868 & 0.9784 & 0.9701 & 0.9619 & 0.9537 \\
\hline 10.00 & 1.0233 & 1.0151 & 1.0067 & 0.9986 & 0.9902 & 0.9820 & 0.9738 & 0.9658 & 0.9578 \\
\hline 15.00 & 1.0261 & 1.0179 & 1.0097 & 1.0018 & 0.9936 & 0.9854 & 0.9775 & 0.9696 & 0.9618 \\
\hline 20.00 & 1.0288 & 1.0208 & 1.0126 & 1.0048 & 0.9968 & 0.9888 & 0.9810 & 0.9732 & 0.9657 \\
\hline 25.00 & 1.0314 & 1.0235 & 1.0155 & 1.0077 & 0.9998 & 0.9920 & 0.9843 & 0.9767 & 0.9694 \\
\hline 30.00 & 1.0340 & 1.0262 & 1.0182 & 1.0106 & 1.0028 & 0.9951 & 0.9876 & 0.9801 & 0.9728 \\
\hline 35.00 & 1.0365 & 1.0288 & 1.0210 & 1.0134 & 1.0058 & 0.9982 & 0.9908 & 0.9834 & 0.9763 \\
\hline 40.00 & 1.0390 & 1.0313 & 1.0236 & 1.0162 & 1.0086 & 1.0011 & 0.9939 & 0.9866 & 0.9797 \\
\hline 45.00 & 1.0414 & 1.0338 & 1.0262 & 1.0189 & 1.0114 & 1.0040 & 0.9969 & 0.9897 & 0.9829 \\
\hline 50.00 & 1.0437 & 1.0363 & 1.0287 & 1.0215 & 1.0141 & 1.0068 & 0.9998 & 0.9928 & 0.9860 \\
\hline 55.00 & 1.0460 & 1.0386 & 1.0312 & 1.0240 & 1.0168 & 1.0095 & 1.0026 & 0.9957 & 0.9890 \\
\hline \multirow[t]{2}{*}{60.00} & 1.0482 & 1.0408 & 1.0335 & 1.0264 & 1.0193 & 1.0121 & 1.0053 & 0.9986 & 0.9919 \\
\hline & & & & & PA & & & & \\
\hline 0.10 & 1.0903 & 1.0805 & 1.0706 & 1.0604 & 1.0504 & 1.0405 & 1.0305 & 1.0204 & 1.0104 \\
\hline 1.00 & 1.0907 & 1.0809 & 1.0712 & 1.0608 & 1.0510 & 1.0411 & 1.0312 & 1.0210 & 1.0111 \\
\hline 5.00 & 1.0929 & 1.0833 & 1.0738 & 1.0635 & 1.0538 & 1.0441 & 1.0343 & 1.0245 & 1.0146 \\
\hline 10.00 & 1.0957 & 1.0862 & 1.0768 & 1.0667 & 1.0573 & 1.0477 & 1.0381 & 1.0285 & 1.0188 \\
\hline 15.00 & 1.0984 & 1.0891 & 1.0797 & 1.0699 & 1.0606 & 1.0512 & 1.0418 & 1.0323 & 1.0229 \\
\hline 20.00 & 1.1011 & 1.0919 & 1.0827 & 1.0729 & 1.0638 & 1.0546 & 1.0453 & 1.0361 & 1.0268 \\
\hline 25.00 & 1.1036 & 1.0946 & 1.0855 & 1.0759 & 1.0669 & 1.0578 & 1.0487 & 1.0397 & 1.0306 \\
\hline 30.00 & 1.1062 & 1.0972 & 1.0883 & 1.0788 & 1.0699 & 1.0610 & 1.0520 & 1.0432 & 1.0342 \\
\hline 35.00 & 1.1087 & 1.0998 & 1.0910 & 1.0817 & 1.0729 & 1.0641 & 1.0553 & 1.0465 & 1.0378 \\
\hline 40.00 & 1.1111 & 1.1024 & 1.0936 & 1.0845 & 1.0758 & 1.0671 & 1.0584 & 1.0499 & 1.0412 \\
\hline 45.00 & 1.1135 & 1.1049 & 1.0962 & 1.0872 & 1.0786 & 1.0701 & 1.0615 & 1.0530 & 1.0445 \\
\hline 50.00 & 1.1158 & 1.1073 & 1.0987 & 1.0898 & 1.0813 & 1.0729 & 1.0644 & 1.0562 & 1.0478 \\
\hline 55.00 & 1.1181 & 1.1097 & 1.1012 & 1.0924 & 1.0840 & 1.0757 & 1.0674 & 1.0592 & 1.0508 \\
\hline \multirow[t]{2}{*}{60.00} & 1.1202 & 1.1119 & 1.1036 & 1.0948 & 1.0866 & 1.0782 & 1.0702 & 1.0621 & 1.0540 \\
\hline & 1.1958 & 1.1862 & 1.1765 & 1.1671 & $\begin{array}{c}\mathrm{MS} \\
1.1573\end{array}$ & 1.1475 & 1.1376 & 1.1274 & 1.1172 \\
\hline 1.00 & 1.1963 & 1.1867 & 1.1771 & 1.1677 & 1.1579 & 1.1481 & 1.1382 & 1.1282 & 1.1180 \\
\hline 5.00 & 1.1985 & 1.1891 & 1.1795 & 1.1702 & 1.1606 & 1.1510 & 1.1412 & 1.1313 & 1.1213 \\
\hline 10.00 & 1.2012 & 1.1919 & 1.1825 & 1.1734 & 1.1639 & 1.1544 & 1.1448 & 1.1351 & 1.1253 \\
\hline 15.00 & 1.2039 & 1.1947 & 1.1854 & 1.1764 & 1.1671 & 1.1578 & 1.1483 & 1.1388 & 1.1292 \\
\hline 20.00 & 1.2065 & 1.1974 & 1.1883 & 1.1794 & 1.1702 & 1.1611 & 1.1517 & 1.1424 & 1.1329 \\
\hline 25.00 & 1.2090 & 1.2001 & 1.1911 & 1.1823 & 1.1732 & 1.1642 & 1.1550 & 1.1458 & 1.1366 \\
\hline 30.00 & 1.2115 & 1.2027 & 1.1938 & 1.1851 & 1.1762 & 1.1673 & 1.1583 & 1.1492 & 1.1401 \\
\hline 35.00 & 1.2140 & 1.2052 & 1.1964 & 1.1879 & 1.1791 & 1.1703 & 1.1614 & 1.1525 & 1.1435 \\
\hline 40.00 & 1.2164 & 1.2077 & 1.1990 & 1.1906 & 1.1819 & 1.1732 & 1.1644 & 1.1556 & 1.1468 \\
\hline 45.00 & 1.2187 & 1.2102 & 1.2016 & 1.1932 & 1.1847 & 1.1761 & 1.1674 & 1.1587 & 1.1500 \\
\hline 50.00 & 1.2210 & 1.2126 & 1.2041 & 1.1959 & 1.1873 & 1.1789 & 1.1704 & 1.1618 & 1.1532 \\
\hline 55.00 & 1.2233 & 1.2150 & 1.2066 & 1.1984 & 1.1900 & 1.1817 & 1.1732 & 1.1647 & 1.1563 \\
\hline 60.00 & 1.2254 & 1.2172 & 1.2089 & 1.2008 & 1.1924 & 1.1842 & 1.1760 & 1.1674 & 1.1593 \\
\hline
\end{tabular}


Table S2. Fitting Parameters of the TRIDEN Correlation of Density $\left(\mathrm{g} \mathrm{cm}^{-3}\right)$ with Pressure and Temperature, eqs. 2 - 4, and AAD values for Pure Solvents. The Parameters are applicable within 278.15 - 358.15 K and $0.1-60$ MPa ranges

\begin{tabular}{ccccccc}
\hline Parameter & $\mathrm{MB}$ & $\mathrm{EB}$ & $\mathrm{PB}$ & $\mathrm{BB}$ & $\mathrm{PA}$ & $\mathrm{MS}$ \\
\hline$C_{T}$ & 0.091502 & 0.083735 & 0.083046 & 0.082852 & 0.086330 & 0.081130 \\
$b_{0}(\mathrm{MPa})$ & 286.484 & 274.841 & 273.127 & 277.275 & 276.393 & 304.543 \\
$b_{1}(\mathrm{MPa})$ & -1.3656 & -23.9139 & -44.8804 & -62.4779 & -9.1679 & 13.7794 \\
$b_{2}(\mathrm{MPa})$ & -17.3213 & -9.5596 & 3.4904 & 13.0039 & -12.3291 & -31.9487 \\
$b_{3}(\mathrm{MPa})$ & 0.49646 & 0.49260 & -1.18874 & -2.30534 & 0.13121 & 3.60509 \\
$E_{T}(\mathrm{~K})$ & 106.45 & 99.26 & 93.59 & 88.99 & 100.97 & 98.47 \\
$A_{R}\left(\mathrm{~g} \mathrm{~cm}{ }^{-3}\right)$ & 45.9026 & 68.3336 & 93.9734 & 131.4046 & 57.0405 & 56.3027 \\
$B_{R}$ & 5.911766 & 7.335465 & 8.707325 & 10.380903 & 6.591603 & 6.312095 \\
$C_{R}(\mathrm{~K})$ & 819.963 & 842.495 & 884.845 & 963.139 & 829.005 & 838.620 \\
$D_{R}$ & -0.228246 & -0.217666 & -0.213754 & -0.219019 & -0.230400 & -0.215635 \\
$\mathrm{AAD}$ & 0.0175 & 0.0138 & 0.0147 & 0.0144 & 0.0164 & 0.0066
\end{tabular}


Table S3. Isobaric Expansivity, $\alpha_{P}\left(\mathrm{kK}^{-1}\right)$ of Pure Solvents as a Function of Pressure and Temperature

\begin{tabular}{|c|c|c|c|c|c|c|c|c|c|}
\hline \multirow{2}{*}{$\begin{array}{c}P \\
(\mathrm{MPa})\end{array}$} & \multicolumn{9}{|c|}{$T(\mathrm{~K})$} \\
\hline & 278.15 & 288.15 & 298.15 & 308.15 & 318.15 & 328.15 & 338.15 & 348.15 & 358.15 \\
\hline & & & & & $\mathrm{MB}$ & & & & \\
\hline 0.10 & 0.823 & 0.842 & 0.862 & 0.882 & 0.904 & 0.927 & 0.950 & 0.975 & 1.001 \\
\hline 1.00 & 0.821 & 0.839 & 0.859 & 0.879 & 0.901 & 0.923 & 0.946 & 0.969 & 0.994 \\
\hline 5.00 & 0.812 & 0.829 & 0.847 & 0.866 & 0.885 & 0.905 & 0.925 & 0.945 & 0.964 \\
\hline 10.00 & 0.801 & 0.817 & 0.834 & 0.850 & 0.867 & 0.884 & 0.900 & 0.915 & 0.929 \\
\hline 15.00 & 0.791 & 0.806 & 0.821 & 0.836 & 0.850 & 0.864 & 0.877 & 0.888 & 0.897 \\
\hline 20.00 & 0.781 & 0.795 & 0.808 & 0.822 & 0.834 & 0.846 & 0.856 & 0.863 & 0.867 \\
\hline 25.00 & 0.771 & 0.784 & 0.796 & 0.808 & 0.819 & 0.828 & 0.836 & 0.840 & 0.839 \\
\hline 30.00 & 0.762 & 0.774 & 0.785 & 0.796 & 0.805 & 0.812 & 0.817 & 0.818 & 0.813 \\
\hline 35.00 & 0.754 & 0.765 & 0.775 & 0.784 & 0.791 & 0.796 & 0.799 & 0.797 & 0.789 \\
\hline 40.00 & 0.746 & 0.755 & 0.764 & 0.772 & 0.778 & 0.782 & 0.782 & 0.777 & 0.766 \\
\hline 45.00 & 0.738 & 0.747 & 0.755 & 0.761 & 0.766 & 0.768 & 0.766 & 0.759 & 0.744 \\
\hline 50.00 & 0.730 & 0.738 & 0.745 & 0.751 & 0.754 & 0.754 & 0.751 & 0.742 & 0.724 \\
\hline 55.00 & 0.723 & 0.730 & 0.736 & 0.741 & 0.743 & 0.742 & 0.736 & 0.725 & 0.705 \\
\hline \multirow[t]{2}{*}{60.00} & 0.716 & 0.723 & 0.728 & 0.731 & 0.732 & 0.730 & 0.723 & 0.709 & 0.687 \\
\hline & & & & & EB & & & & \\
\hline 0.10 & 0.839 & 0.857 & 0.876 & 0.896 & 0.917 & 0.939 & 0.962 & 0.985 & 1.010 \\
\hline 1.00 & 0.836 & 0.854 & 0.873 & 0.893 & 0.913 & 0.934 & 0.956 & 0.979 & 1.003 \\
\hline 5.00 & 0.826 & 0.843 & 0.860 & 0.878 & 0.897 & 0.915 & 0.934 & 0.953 & 0.972 \\
\hline 10.00 & 0.814 & 0.829 & 0.845 & 0.861 & 0.877 & 0.893 & 0.909 & 0.924 & 0.937 \\
\hline 15.00 & 0.802 & 0.816 & 0.831 & 0.845 & 0.859 & 0.872 & 0.885 & 0.896 & 0.905 \\
\hline 20.00 & 0.791 & 0.804 & 0.817 & 0.830 & 0.842 & 0.853 & 0.863 & 0.871 & 0.876 \\
\hline 25.00 & 0.781 & 0.793 & 0.805 & 0.816 & 0.826 & 0.835 & 0.843 & 0.848 & 0.849 \\
\hline 30.00 & 0.771 & 0.782 & 0.793 & 0.802 & 0.811 & 0.819 & 0.824 & 0.826 & 0.824 \\
\hline 35.00 & 0.762 & 0.772 & 0.781 & 0.790 & 0.797 & 0.803 & 0.806 & 0.806 & 0.801 \\
\hline 40.00 & 0.753 & 0.762 & 0.771 & 0.778 & 0.784 & 0.788 & 0.790 & 0.787 & 0.780 \\
\hline 45.00 & 0.744 & 0.753 & 0.760 & 0.767 & 0.772 & 0.774 & 0.774 & 0.770 & 0.760 \\
\hline 50.00 & 0.736 & 0.744 & 0.751 & 0.756 & 0.760 & 0.761 & 0.760 & 0.753 & 0.741 \\
\hline 55.00 & 0.729 & 0.736 & 0.742 & 0.746 & 0.749 & 0.749 & 0.746 & 0.738 & 0.724 \\
\hline \multirow[t]{2}{*}{60.00} & 0.721 & 0.728 & 0.733 & 0.737 & 0.738 & 0.737 & 0.733 & 0.723 & 0.708 \\
\hline & & & & & PB & & & & \\
\hline 0.10 & 0.827 & 0.843 & 0.861 & 0.879 & 0.898 & 0.918 & 0.938 & 0.959 & 0.981 \\
\hline 1.00 & 0.824 & 0.841 & 0.858 & 0.876 & 0.894 & 0.913 & 0.933 & 0.953 & 0.974 \\
\hline 5.00 & 0.815 & 0.830 & 0.846 & 0.862 & 0.878 & 0.894 & 0.911 & 0.927 & 0.942 \\
\hline 10.00 & 0.803 & 0.817 & 0.831 & 0.845 & 0.859 & 0.872 & 0.885 & 0.896 & 0.906 \\
\hline 15.00 & 0.792 & 0.805 & 0.817 & 0.830 & 0.841 & 0.852 & 0.862 & 0.869 & 0.873 \\
\hline 20.00 & 0.782 & 0.793 & 0.805 & 0.815 & 0.825 & 0.834 & 0.840 & 0.843 & 0.842 \\
\hline 25.00 & 0.772 & 0.783 & 0.793 & 0.802 & 0.810 & 0.816 & 0.820 & 0.820 & 0.815 \\
\hline 30.00 & 0.763 & 0.773 & 0.781 & 0.789 & 0.796 & 0.800 & 0.801 & 0.798 & 0.789 \\
\hline 35.00 & 0.754 & 0.763 & 0.771 & 0.777 & 0.782 & 0.784 & 0.784 & 0.778 & 0.765 \\
\hline 40.00 & 0.746 & 0.754 & 0.761 & 0.766 & 0.769 & 0.770 & 0.767 & 0.759 & 0.743 \\
\hline 45.00 & 0.738 & 0.745 & 0.751 & 0.755 & 0.757 & 0.757 & 0.752 & 0.741 & 0.723 \\
\hline 50.00 & 0.731 & 0.737 & 0.742 & 0.745 & 0.746 & 0.744 & 0.737 & 0.725 & 0.704 \\
\hline 55.00 & 0.724 & 0.729 & 0.733 & 0.736 & 0.736 & 0.732 & 0.724 & 0.709 & 0.686 \\
\hline 60.00 & 0.717 & 0.722 & 0.725 & 0.727 & 0.725 & 0.721 & 0.711 & 0.695 & 0.669 \\
\hline
\end{tabular}


Table S3. Continued

\begin{tabular}{|c|c|c|c|c|c|c|c|c|c|}
\hline \multirow[b]{2}{*}{$\begin{array}{c}P \\
(\mathrm{MPa})\end{array}$} & \multicolumn{9}{|c|}{$T(\mathrm{~K})$} \\
\hline & 278.15 & 288.15 & 298.15 & 308.15 & 318.15 & 328.15 & 338.15 & 348.15 & 358.15 \\
\hline & & & & & BB & & & & \\
\hline 0.10 & 0.806 & 0.821 & 0.836 & 0.851 & 0.868 & 0.884 & 0.901 & 0.919 & 0.938 \\
\hline 1.00 & 0.804 & 0.818 & 0.833 & 0.848 & 0.864 & 0.880 & 0.896 & 0.913 & 0.930 \\
\hline 5.00 & 0.795 & 0.808 & 0.821 & 0.835 & 0.848 & 0.862 & 0.874 & 0.887 & 0.897 \\
\hline 10.00 & 0.784 & 0.796 & 0.808 & 0.819 & 0.830 & 0.840 & 0.849 & 0.856 & 0.860 \\
\hline 15.00 & 0.774 & 0.785 & 0.795 & 0.804 & 0.813 & 0.820 & 0.826 & 0.828 & 0.825 \\
\hline 20.00 & 0.765 & 0.774 & 0.783 & 0.791 & 0.797 & 0.802 & 0.804 & 0.802 & 0.794 \\
\hline 25.00 & 0.756 & 0.764 & 0.772 & 0.778 & 0.783 & 0.785 & 0.784 & 0.778 & 0.766 \\
\hline 30.00 & 0.747 & 0.755 & 0.761 & 0.766 & 0.769 & 0.769 & 0.765 & 0.756 & 0.739 \\
\hline 35.00 & 0.739 & 0.746 & 0.751 & 0.754 & 0.756 & 0.754 & 0.748 & 0.736 & 0.715 \\
\hline 40.00 & 0.732 & 0.737 & 0.741 & 0.744 & 0.744 & 0.740 & 0.732 & 0.717 & 0.692 \\
\hline 45.00 & 0.724 & 0.729 & 0.732 & 0.733 & 0.732 & 0.727 & 0.716 & 0.699 & 0.671 \\
\hline 50.00 & 0.717 & 0.721 & 0.724 & 0.724 & 0.721 & 0.714 & 0.702 & 0.682 & 0.651 \\
\hline 55.00 & 0.711 & 0.714 & 0.716 & 0.715 & 0.711 & 0.703 & 0.688 & 0.666 & 0.632 \\
\hline \multirow[t]{2}{*}{60.00} & 0.704 & 0.707 & 0.708 & 0.706 & 0.701 & 0.691 & 0.676 & 0.651 & 0.615 \\
\hline & & & & & PA & & & & \\
\hline 0.10 & 0.867 & 0.886 & 0.907 & 0.928 & 0.951 & 0.974 & 0.999 & 1.024 & 1.051 \\
\hline 1.00 & 0.864 & 0.884 & 0.904 & 0.925 & 0.947 & 0.970 & 0.993 & 1.018 & 1.043 \\
\hline 5.00 & 0.855 & 0.873 & 0.892 & 0.911 & 0.931 & 0.951 & 0.971 & 0.991 & 1.010 \\
\hline 10.00 & 0.844 & 0.860 & 0.877 & 0.894 & 0.912 & 0.928 & 0.945 & 0.960 & 0.972 \\
\hline 15.00 & 0.833 & 0.848 & 0.864 & 0.879 & 0.894 & 0.908 & 0.920 & 0.931 & 0.937 \\
\hline 20.00 & 0.823 & 0.837 & 0.851 & 0.864 & 0.877 & 0.888 & 0.897 & 0.904 & 0.905 \\
\hline 25.00 & 0.813 & 0.826 & 0.838 & 0.850 & 0.861 & 0.870 & 0.876 & 0.879 & 0.875 \\
\hline 30.00 & 0.804 & 0.816 & 0.827 & 0.837 & 0.846 & 0.853 & 0.856 & 0.855 & 0.848 \\
\hline 35.00 & 0.795 & 0.806 & 0.816 & 0.825 & 0.832 & 0.837 & 0.838 & 0.834 & 0.822 \\
\hline 40.00 & 0.786 & 0.796 & 0.805 & 0.813 & 0.818 & 0.821 & 0.820 & 0.813 & 0.798 \\
\hline 45.00 & 0.778 & 0.787 & 0.795 & 0.802 & 0.806 & 0.807 & 0.804 & 0.794 & 0.776 \\
\hline 50.00 & 0.771 & 0.779 & 0.786 & 0.791 & 0.794 & 0.793 & 0.788 & 0.776 & 0.755 \\
\hline 55.00 & 0.763 & 0.771 & 0.777 & 0.781 & 0.782 & 0.780 & 0.773 & 0.759 & 0.735 \\
\hline \multirow[t]{2}{*}{60.00} & 0.756 & 0.763 & 0.768 & 0.771 & 0.771 & 0.768 & 0.759 & 0.743 & 0.716 \\
\hline & 0.773 & 0.790 & 0.808 & 0.827 & $\begin{array}{c}\mathrm{MS} \\
0.846\end{array}$ & 0.866 & 0.887 & 0.909 & 0.932 \\
\hline 1.00 & 0.771 & 0.788 & 0.806 & 0.824 & 0.843 & 0.863 & 0.883 & 0.905 & 0.927 \\
\hline 5.00 & 0.762 & 0.778 & 0.795 & 0.812 & 0.830 & 0.848 & 0.867 & 0.886 & 0.906 \\
\hline 10.00 & 0.752 & 0.767 & 0.782 & 0.798 & 0.814 & 0.830 & 0.847 & 0.864 & 0.882 \\
\hline 15.00 & 0.742 & 0.755 & 0.770 & 0.784 & 0.799 & 0.814 & 0.829 & 0.844 & 0.859 \\
\hline 20.00 & 0.732 & 0.745 & 0.758 & 0.771 & 0.785 & 0.798 & 0.812 & 0.825 & 0.838 \\
\hline 25.00 & 0.723 & 0.735 & 0.747 & 0.759 & 0.772 & 0.784 & 0.795 & 0.807 & 0.818 \\
\hline 30.00 & 0.714 & 0.725 & 0.737 & 0.748 & 0.759 & 0.770 & 0.780 & 0.790 & 0.800 \\
\hline 35.00 & 0.706 & 0.716 & 0.727 & 0.737 & 0.747 & 0.757 & 0.766 & 0.775 & 0.782 \\
\hline 40.00 & 0.698 & 0.708 & 0.717 & 0.727 & 0.736 & 0.745 & 0.753 & 0.760 & 0.766 \\
\hline 45.00 & 0.690 & 0.699 & 0.708 & 0.717 & 0.725 & 0.733 & 0.740 & 0.746 & 0.751 \\
\hline 50.00 & 0.683 & 0.691 & 0.700 & 0.708 & 0.715 & 0.722 & 0.728 & 0.733 & 0.737 \\
\hline 55.00 & 0.676 & 0.684 & 0.691 & 0.699 & 0.705 & 0.711 & 0.717 & 0.721 & 0.724 \\
\hline 60.00 & 0.669 & 0.676 & 0.684 & 0.690 & 0.696 & 0.701 & 0.706 & 0.709 & 0.711 \\
\hline
\end{tabular}


Table S4 Isothermal Compressibility, $\kappa_{T}\left(\mathrm{TPa}^{-1}\right)$, of Pure Solvents as a Function of Pressure and Temperature

\begin{tabular}{|c|c|c|c|c|c|c|c|c|c|}
\hline \multirow[b]{2}{*}{$\begin{array}{c}P \\
(\mathrm{MPa})\end{array}$} & \multicolumn{9}{|c|}{$T(\mathrm{~K})$} \\
\hline & 278.15 & 288.15 & 298.15 & 308.15 & 318.15 & 328.15 & 338.15 & 348.15 & 358.15 \\
\hline & & & & & $\mathrm{MB}$ & & & & \\
\hline 0.10 & 527.1 & 551.8 & 579.9 & 611.9 & 648.8 & 691.5 & 741.7 & 801.2 & 872.9 \\
\hline 1.00 & 524.6 & 549.1 & 576.9 & 608.6 & 645.0 & 687.3 & 736.8 & 795.5 & 866.1 \\
\hline 5.00 & 513.9 & 537.4 & 564.0 & 594.3 & 629.0 & 669.1 & 715.9 & 771.2 & 837.4 \\
\hline 10.00 & 501.2 & 523.5 & 548.7 & 577.3 & 610.0 & 647.7 & 691.4 & 742.9 & 804.2 \\
\hline 15.00 & 489.1 & 510.3 & 534.3 & 561.4 & 592.2 & 627.7 & 668.7 & 716.8 & 773.7 \\
\hline 20.00 & 477.6 & 497.9 & 520.6 & 546.3 & 575.5 & 608.9 & 647.5 & 692.5 & 745.5 \\
\hline 25.00 & 466.7 & 486.0 & 507.7 & 532.1 & 559.8 & 591.3 & 627.7 & 669.9 & 719.4 \\
\hline 30.00 & 456.3 & 474.7 & 495.4 & 518.6 & 544.9 & 574.8 & 609.1 & 648.7 & 695.1 \\
\hline 35.00 & 446.3 & 464.0 & 483.7 & 505.9 & 530.8 & 559.2 & 591.6 & 629.0 & 672.6 \\
\hline 40.00 & 436.8 & 453.7 & 472.6 & 493.7 & 517.5 & 544.4 & 575.2 & 610.5 & 651.5 \\
\hline 45.00 & 427.8 & 444.0 & 462.0 & 482.2 & 504.9 & 530.5 & 559.7 & 593.1 & 631.7 \\
\hline 50.00 & 419.1 & 434.6 & 451.9 & 471.2 & 492.9 & 517.3 & 545.0 & 576.7 & 613.2 \\
\hline 55.00 & 410.8 & 425.7 & 442.3 & 460.8 & 481.5 & 504.8 & 531.2 & 561.2 & 595.8 \\
\hline \multirow[t]{2}{*}{60.00} & 402.8 & 417.2 & 433.1 & 450.8 & 470.6 & 492.9 & 518.0 & 546.6 & 579.4 \\
\hline & & & & & EB & & & & \\
\hline 0.10 & 582.7 & 611.2 & 643.1 & 679.1 & 720.0 & 766.8 & 820.9 & 884.0 & 958.7 \\
\hline 1.00 & 579.4 & 607.5 & 639.0 & 674.6 & 714.9 & 761.0 & 814.3 & 876.4 & 949.7 \\
\hline 5.00 & 565.1 & 591.8 & 621.7 & 655.3 & 693.2 & 736.6 & 786.3 & 844.1 & 912.0 \\
\hline 10.00 & 548.2 & 573.3 & 601.3 & 632.7 & 668.1 & 708.2 & 754.1 & 807.1 & 868.9 \\
\hline 15.00 & 532.4 & 556.0 & 582.3 & 611.7 & 644.7 & 682.0 & 724.5 & 773.3 & 829.9 \\
\hline 20.00 & 517.4 & 539.8 & 564.5 & 592.1 & 623.0 & 657.8 & 697.3 & 742.4 & 794.5 \\
\hline 25.00 & 503.4 & 524.5 & 547.9 & 573.8 & 602.8 & 635.3 & 672.1 & 714.0 & 762.0 \\
\hline 30.00 & 490.1 & 510.1 & 532.2 & 556.7 & 583.9 & 614.4 & 648.8 & 687.7 & 732.3 \\
\hline 35.00 & 477.5 & 496.5 & 517.4 & 540.5 & 566.2 & 594.9 & 627.1 & 663.4 & 704.8 \\
\hline 40.00 & 465.6 & 483.7 & 503.5 & 525.4 & 549.6 & 576.6 & 606.8 & 640.9 & 679.5 \\
\hline 45.00 & 454.3 & 471.5 & 490.3 & 511.1 & 534.0 & 559.5 & 587.9 & 619.9 & 656.0 \\
\hline 50.00 & 443.6 & 460.0 & 477.9 & 497.6 & 519.3 & 543.4 & 570.2 & 600.3 & 634.1 \\
\hline 55.00 & 433.4 & 449.0 & 466.1 & 484.8 & 505.5 & 528.3 & 553.6 & 581.9 & 613.7 \\
\hline \multirow[t]{2}{*}{60.00} & 423.6 & 438.6 & 454.9 & 472.7 & 492.3 & 514.0 & 538.0 & 564.7 & 594.7 \\
\hline & & & & & PB & & & & \\
\hline 0.10 & 595.5 & 622.4 & 652.7 & 687.1 & 726.6 & 772.3 & 825.8 & 889.4 & 966.0 \\
\hline 1.00 & 592.0 & 618.5 & 648.5 & 682.5 & 721.4 & 766.4 & 819.1 & 881.6 & 956.8 \\
\hline 5.00 & 576.9 & 602.1 & 630.5 & 662.5 & 699.2 & 741.4 & 790.6 & 848.6 & 918.1 \\
\hline 10.00 & 559.2 & 582.8 & 609.4 & 639.3 & 673.3 & 712.4 & 757.7 & 810.9 & 874.1 \\
\hline 15.00 & 542.6 & 564.8 & 589.7 & 617.7 & 649.4 & 685.7 & 727.6 & 776.5 & 834.4 \\
\hline 20.00 & 526.9 & 547.9 & 571.3 & 597.5 & 627.2 & 661.0 & 699.9 & 745.1 & 798.2 \\
\hline 25.00 & 512.2 & 532.0 & 554.1 & 578.7 & 606.5 & 638.1 & 674.3 & 716.2 & 765.2 \\
\hline 30.00 & 498.4 & 517.1 & 537.9 & 561.1 & 587.3 & 616.8 & 650.6 & 689.6 & 734.9 \\
\hline 35.00 & 485.3 & 503.0 & 522.7 & 544.6 & 569.2 & 597.0 & 628.6 & 664.9 & 707.1 \\
\hline 40.00 & 472.9 & 489.7 & 508.4 & 529.1 & 552.3 & 578.4 & 608.1 & 642.1 & 681.3 \\
\hline 45.00 & 461.1 & 477.2 & 494.9 & 514.5 & 536.4 & 561.1 & 589.0 & 620.8 & 657.5 \\
\hline 50.00 & 450.0 & 465.2 & 482.1 & 500.7 & 521.5 & 544.8 & 571.0 & 601.0 & 635.4 \\
\hline 55.00 & 439.4 & 453.9 & 469.9 & 487.7 & 507.4 & 529.4 & 554.2 & 582.4 & 614.7 \\
\hline 60.00 & 429.3 & 443.2 & 458.5 & 475.3 & 494.0 & 514.9 & 538.4 & 565.1 & 595.5 \\
\hline
\end{tabular}


Table S4. Continued

\begin{tabular}{|c|c|c|c|c|c|c|c|c|c|}
\hline \multirow[b]{2}{*}{$\begin{array}{c}P \\
(\mathrm{MPa})\end{array}$} & \multicolumn{9}{|c|}{$T(\mathrm{~K})$} \\
\hline & 278.15 & 288.15 & 298.15 & 308.15 & 318.15 & 328.15 & 338.15 & 348.15 & 358.15 \\
\hline & & & & & BB & & & & \\
\hline 0.10 & 597.2 & 622.3 & 650.7 & 683.4 & 721.2 & 765.6 & 818.3 & 882.0 & 960.3 \\
\hline 1.00 & 593.6 & 618.4 & 646.5 & 678.8 & 716.1 & 759.8 & 811.7 & 874.3 & 951.2 \\
\hline 5.00 & 578.5 & 602.0 & 628.6 & 659.0 & 694.1 & 735.1 & 783.6 & 841.8 & 912.9 \\
\hline 10.00 & 560.6 & 582.7 & 607.6 & 635.9 & 668.6 & 706.6 & 751.2 & 804.6 & 869.3 \\
\hline 15.00 & 543.9 & 564.6 & 588.0 & 614.5 & 644.9 & 680.2 & 721.6 & 770.6 & 829.9 \\
\hline 20.00 & 528.1 & 547.7 & 569.6 & 594.5 & 623.0 & 655.9 & 694.2 & 739.6 & 794.0 \\
\hline 25.00 & 513.3 & 531.8 & 552.5 & 575.9 & 602.5 & 633.3 & 669.0 & 711.0 & 761.2 \\
\hline 30.00 & 499.4 & 516.8 & 536.4 & 558.4 & 583.5 & 612.2 & 645.6 & 684.7 & 731.2 \\
\hline 35.00 & 486.2 & 502.7 & 521.2 & 542.0 & 565.6 & 592.6 & 623.9 & 660.4 & 703.5 \\
\hline 40.00 & 473.7 & 489.4 & 506.9 & 526.6 & 548.9 & 574.3 & 603.6 & 637.8 & 678.0 \\
\hline 45.00 & 461.9 & 476.8 & 493.4 & 512.1 & 533.1 & 557.1 & 584.7 & 616.7 & 654.3 \\
\hline 50.00 & 450.7 & 464.9 & 480.7 & 498.4 & 518.3 & 541.0 & 567.0 & 597.1 & 632.3 \\
\hline 55.00 & 440.0 & 453.6 & 468.6 & 485.4 & 504.3 & 525.8 & 550.4 & 578.7 & 611.8 \\
\hline \multirow[t]{2}{*}{60.00} & 429.9 & 442.8 & 457.1 & 473.1 & 491.1 & 511.5 & 534.7 & 561.5 & 592.7 \\
\hline & 538.2 & 564.4 & 594.2 & 628.3 & $\begin{array}{c}\text { PA } \\
667.7\end{array}$ & 713.6 & 767.9 & 832.7 & 911.6 \\
\hline 1.00 & 535.4 & 561.4 & 590.8 & 624.6 & 663.5 & 708.8 & 762.3 & 826.2 & 903.8 \\
\hline 5.00 & 523.6 & 548.4 & 576.4 & 608.5 & 645.4 & 688.2 & 738.5 & 798.3 & 870.6 \\
\hline 10.00 & 509.5 & 533.0 & 559.5 & 589.6 & 624.2 & 664.2 & 710.9 & 766.2 & 832.5 \\
\hline 15.00 & 496.2 & 518.4 & 543.5 & 571.9 & 604.4 & 641.8 & 685.4 & 736.6 & 797.7 \\
\hline 20.00 & 483.7 & 504.7 & 528.5 & 555.3 & 585.9 & 621.0 & 661.7 & 709.4 & 765.9 \\
\hline 25.00 & 471.7 & 491.8 & 514.3 & 539.7 & 568.5 & 601.5 & 639.7 & 684.2 & 736.7 \\
\hline 30.00 & 460.4 & 479.5 & 500.9 & 524.9 & 552.2 & 583.3 & 619.2 & 660.8 & 709.7 \\
\hline 35.00 & 449.6 & 467.8 & 488.2 & 511.0 & 536.8 & 566.2 & 600.0 & 639.0 & 684.7 \\
\hline 40.00 & 439.4 & 456.8 & 476.1 & 497.9 & 522.4 & 550.2 & 582.0 & 618.7 & 661.5 \\
\hline 45.00 & 429.6 & 446.2 & 464.7 & 485.4 & 508.7 & 535.0 & 565.1 & 599.7 & 639.8 \\
\hline 50.00 & 420.3 & 436.2 & 453.8 & 473.6 & 495.7 & 520.8 & 549.2 & 581.9 & 619.7 \\
\hline 55.00 & 411.4 & 426.6 & 443.5 & 462.3 & 483.5 & 507.3 & 534.3 & 565.2 & 600.8 \\
\hline \multirow[t]{2}{*}{60.00} & 402.9 & 417.5 & 433.6 & 451.7 & 471.8 & 494.5 & 520.1 & 549.4 & 583.1 \\
\hline & 477.5 & 501.7 & 528.4 & 558.1 & $\begin{array}{c}\mathrm{MS} \\
591.2\end{array}$ & 628.1 & 669.4 & 716.0 & 7685 \\
\hline 1.00 & 475.2 & 499.1 & 525.6 & 555.0 & 587.6 & 624.1 & 664.9 & 710.8 & 762.5 \\
\hline 5.00 & 465.2 & 488.1 & 513.4 & 541.4 & 572.4 & 607.0 & 645.5 & 688.7 & 737.1 \\
\hline 10.00 & 453.3 & 475.0 & 498.9 & 525.3 & 554.5 & 586.9 & 622.9 & 662.9 & 707.8 \\
\hline 15.00 & 442.0 & 462.7 & 485.3 & 510.3 & 537.8 & 568.2 & 601.8 & 639.2 & 680.7 \\
\hline 20.00 & 431.3 & 451.0 & 472.5 & 496.1 & 522.0 & 550.6 & 582.2 & 617.1 & 655.8 \\
\hline 25.00 & 421.2 & 439.9 & 460.3 & 482.7 & 507.2 & 534.2 & 563.9 & 596.6 & 632.7 \\
\hline 30.00 & 411.5 & 429.3 & 448.8 & 470.0 & 493.3 & 518.8 & 546.7 & 577.5 & 611.2 \\
\hline 35.00 & 402.2 & 419.3 & 437.8 & 458.1 & 480.1 & 504.3 & 530.7 & 559.6 & 591.3 \\
\hline 40.00 & 393.4 & 409.7 & 427.4 & 446.7 & 467.7 & 490.6 & 515.5 & 542.8 & 572.6 \\
\hline 45.00 & 385.0 & 400.6 & 417.5 & 435.9 & 455.9 & 477.6 & 501.3 & 527.1 & 555.1 \\
\hline 50.00 & 377.0 & 391.9 & 408.1 & 425.7 & 444.7 & 465.4 & 487.8 & 512.2 & 538.8 \\
\hline 55.00 & 369.3 & 383.6 & 399.1 & 415.9 & 434.1 & 453.8 & 475.1 & 498.3 & 523.4 \\
\hline 60.00 & 361.9 & 375.7 & 390.6 & 406.6 & 424.0 & 442.8 & 463.1 & 485.1 & 508.9 \\
\hline
\end{tabular}


Table S5. Internal Pressure, $P_{i}(\mathrm{MPa})$, of Pure Solvents as a Function of Pressure and Temperature

\begin{tabular}{|c|c|c|c|c|c|c|c|c|c|}
\hline \multirow[b]{2}{*}{$\begin{array}{c}P \\
(\mathrm{MPa})\end{array}$} & \multicolumn{9}{|c|}{$T(\mathrm{~K})$} \\
\hline & 278.15 & 288.15 & 298.15 & 308.15 & 318.15 & 328.15 & 338.15 & 348.15 & 358.15 \\
\hline & & & & & $\mathrm{MB}$ & & & & \\
\hline 0.10 & 434.1 & 439.5 & 442.9 & 444.3 & 443.2 & 439.7 & 433.2 & 423.7 & 410.7 \\
\hline 1.00 & 434.2 & 439.5 & 442.9 & 444.2 & 443.2 & 439.5 & 433.0 & 423.3 & 410.1 \\
\hline 5.00 & 434.3 & 439.6 & 443.0 & 444.1 & 442.8 & 438.8 & 431.8 & 421.4 & 407.3 \\
\hline 10.00 & 434.5 & 439.8 & 443.0 & 443.9 & 442.3 & 437.9 & 430.2 & 419.0 & 403.8 \\
\hline 15.00 & 434.6 & 439.8 & 442.9 & 443.7 & 441.8 & 436.9 & 428.6 & 416.6 & 400.2 \\
\hline 20.00 & 434.7 & 439.9 & 442.9 & 443.4 & 441.2 & 435.8 & 427.0 & 414.0 & 396.5 \\
\hline 25.00 & 434.8 & 439.9 & 442.8 & 443.1 & 440.5 & 434.7 & 425.2 & 411.5 & 392.7 \\
\hline 30.00 & 434.8 & 439.9 & 442.6 & 442.7 & 439.8 & 433.6 & 423.5 & 408.8 & 388.9 \\
\hline 35.00 & 434.9 & 439.8 & 442.4 & 442.3 & 439.1 & 432.4 & 421.6 & 406.1 & 385.0 \\
\hline 40.00 & 434.9 & 439.8 & 442.2 & 441.9 & 438.4 & 431.2 & 419.7 & 403.3 & 381.0 \\
\hline 45.00 & 434.8 & 439.6 & 442.0 & 441.4 & 437.6 & 429.9 & 417.8 & 400.5 & 377.0 \\
\hline 50.00 & 434.8 & 439.5 & 441.7 & 440.9 & 436.7 & 428.6 & 415.8 & 397.7 & 372.9 \\
\hline 55.00 & 434.7 & 439.3 & 441.4 & 440.4 & 435.8 & 427.2 & 413.8 & 394.7 & 368.7 \\
\hline \multirow[t]{2}{*}{60.00} & 434.5 & 439.2 & 441.0 & 439.8 & 434.9 & 425.8 & 411.8 & 391.8 & 364.5 \\
\hline & & & & & EB & & & & \\
\hline 0.10 & 400.2 & 404.0 & 406.2 & 406.6 & 405.2 & 401.7 & 396.0 & 388.0 & 377.3 \\
\hline 1.00 & 400.5 & 404.3 & 406.4 & 406.9 & 405.4 & 401.9 & 396.2 & 388.0 & 377.2 \\
\hline 5.00 & 401.5 & 405.4 & 407.6 & 408.0 & 406.5 & 402.8 & 396.8 & 388.2 & 376.8 \\
\hline 10.00 & 402.8 & 406.7 & 409.0 & 409.3 & 407.7 & 403.8 & 397.4 & 388.4 & 376.3 \\
\hline 15.00 & 404.0 & 408.0 & 410.3 & 410.6 & 408.9 & 404.7 & 398.0 & 388.5 & 375.6 \\
\hline 20.00 & 405.2 & 409.3 & 411.6 & 411.9 & 410.0 & 405.6 & 398.6 & 388.5 & 374.9 \\
\hline 25.00 & 406.4 & 410.5 & 412.8 & 413.1 & 411.0 & 406.5 & 399.0 & 388.4 & 374.0 \\
\hline 30.00 & 407.5 & 411.7 & 414.0 & 414.2 & 412.0 & 407.2 & 399.4 & 388.2 & 373.1 \\
\hline 35.00 & 408.7 & 412.9 & 415.2 & 415.3 & 413.0 & 407.9 & 399.8 & 388.0 & 372.1 \\
\hline 40.00 & 409.7 & 414.0 & 416.3 & 416.4 & 413.9 & 408.6 & 400.1 & 387.7 & 371.1 \\
\hline 45.00 & 410.8 & 415.1 & 417.4 & 417.4 & 414.8 & 409.2 & 400.3 & 387.4 & 369.9 \\
\hline 50.00 & 411.8 & 416.1 & 418.4 & 418.4 & 415.6 & 409.8 & 400.4 & 387.0 & 368.7 \\
\hline 55.00 & 412.7 & 417.2 & 419.4 & 419.3 & 416.4 & 410.3 & 400.6 & 386.5 & 367.5 \\
\hline \multirow[t]{2}{*}{60.00} & 413.7 & 418.2 & 420.4 & 420.2 & 417.1 & 410.8 & 400.6 & 386.0 & 366.1 \\
\hline & & & & & PB & & & & \\
\hline 0.10 & 386.0 & 390.4 & 393.2 & 394.1 & 393.1 & 389.7 & 384.0 & 375.4 & 363.7 \\
\hline 1.00 & 386.3 & 390.7 & 393.5 & 394.4 & 393.3 & 390.0 & 384.1 & 375.4 & 363.5 \\
\hline 5.00 & 387.7 & 392.1 & 394.9 & 395.8 & 394.5 & 390.8 & 384.5 & 375.2 & 362.5 \\
\hline 10.00 & 389.4 & 393.9 & 396.6 & 397.4 & 395.9 & 391.9 & 385.0 & 374.9 & 361.0 \\
\hline 15.00 & 391.1 & 395.6 & 398.3 & 399.0 & 397.2 & 392.9 & 385.4 & 374.5 & 359.5 \\
\hline 20.00 & 392.7 & 397.3 & 400.0 & 400.5 & 398.5 & 393.8 & 385.8 & 374.1 & 358.0 \\
\hline 25.00 & 394.3 & 398.9 & 401.6 & 402.0 & 399.8 & 394.7 & 386.1 & 373.6 & 356.3 \\
\hline 30.00 & 395.9 & 400.5 & 403.1 & 403.4 & 401.0 & 395.5 & 386.3 & 373.0 & 354.5 \\
\hline 35.00 & 397.4 & 402.1 & 404.6 & 404.8 & 402.1 & 396.2 & 386.5 & 372.3 & 352.7 \\
\hline 40.00 & 398.9 & 403.6 & 406.1 & 406.1 & 403.2 & 396.9 & 386.6 & 371.6 & 350.8 \\
\hline 45.00 & 400.3 & 405.1 & 407.5 & 407.4 & 404.3 & 397.6 & 386.7 & 370.8 & 348.8 \\
\hline 50.00 & 401.8 & 406.5 & 408.9 & 408.7 & 405.3 & 398.2 & 386.7 & 369.9 & 346.8 \\
\hline 55.00 & 403.2 & 407.9 & 410.3 & 409.9 & 406.2 & 398.7 & 386.6 & 369.0 & 344.7 \\
\hline 60.00 & 404.5 & 409.3 & 411.6 & 411.1 & 407.1 & 399.2 & 386.5 & 368.1 & 342.5 \\
\hline
\end{tabular}


Table S5. Continued

\begin{tabular}{|c|c|c|c|c|c|c|c|c|c|}
\hline \multirow[b]{2}{*}{$\begin{array}{c}P \\
(\mathrm{MPa})\end{array}$} & \multicolumn{9}{|c|}{$T(\mathrm{~K})$} \\
\hline & 278.15 & 288.15 & 298.15 & 308.15 & 318.15 & 328.15 & 338.15 & 348.15 & 358.15 \\
\hline & & & & & BB & & & & \\
\hline 0.10 & 375.4 & 380.0 & 382.9 & 383.8 & 382.6 & 378.9 & 372.4 & 362.8 & 349.7 \\
\hline 1.00 & 375.7 & 380.3 & 383.2 & 384.1 & 382.8 & 379.0 & 372.4 & 362.6 & 349.2 \\
\hline 5.00 & 377.3 & 381.8 & 384.6 & 385.4 & 383.8 & 379.6 & 372.3 & 361.6 & 347.0 \\
\hline 10.00 & 379.1 & 383.7 & 386.4 & 386.9 & 385.0 & 380.2 & 372.2 & 360.4 & 344.2 \\
\hline 15.00 & 380.9 & 385.5 & 388.1 & 388.4 & 386.1 & 380.8 & 372.0 & 359.0 & 341.3 \\
\hline 20.00 & 382.7 & 387.3 & 389.7 & 389.8 & 387.2 & 381.3 & 371.7 & 357.6 & 338.3 \\
\hline 25.00 & 384.5 & 389.0 & 391.4 & 391.2 & 388.2 & 381.8 & 371.3 & 356.1 & 335.2 \\
\hline 30.00 & 386.2 & 390.7 & 392.9 & 392.6 & 389.2 & 382.2 & 370.9 & 354.6 & 332.0 \\
\hline 35.00 & 387.9 & 392.3 & 394.5 & 393.9 & 390.1 & 382.5 & 370.4 & 352.9 & 328.8 \\
\hline 40.00 & 389.5 & 394.0 & 396.0 & 395.2 & 391.0 & 382.8 & 369.9 & 351.3 & 325.5 \\
\hline 45.00 & 391.2 & 395.6 & 397.4 & 396.4 & 391.8 & 383.1 & 369.3 & 349.5 & 322.1 \\
\hline 50.00 & 392.7 & 397.1 & 398.9 & 397.6 & 392.6 & 383.3 & 368.7 & 347.7 & 318.7 \\
\hline 55.00 & 394.3 & 398.6 & 400.3 & 398.7 & 393.3 & 383.4 & 368.0 & 345.8 & 315.2 \\
\hline \multirow[t]{2}{*}{60.00} & 395.8 & 400.1 & 401.6 & 399.8 & 394.1 & 383.6 & 367.3 & 343.9 & 311.7 \\
\hline & & & & & PA & & & & \\
\hline 0.10 & 447.8 & 452.5 & 455.0 & 455.3 & 453.0 & 447.9 & 439.7 & 428.2 & 412.9 \\
\hline 1.00 & 448.1 & 452.7 & 455.3 & 455.5 & 453.2 & 448.0 & 439.7 & 428.0 & 412.5 \\
\hline 5.00 & 449.2 & 453.8 & 456.3 & 456.4 & 453.8 & 448.3 & 439.6 & 427.2 & 410.6 \\
\hline 10.00 & 450.5 & 455.1 & 457.6 & 457.5 & 454.7 & 448.7 & 439.3 & 426.0 & 408.3 \\
\hline 15.00 & 451.8 & 456.4 & 458.8 & 458.5 & 455.4 & 449.1 & 439.0 & 424.8 & 405.8 \\
\hline 20.00 & 453.1 & 457.7 & 459.9 & 459.5 & 456.1 & 449.3 & 438.6 & 423.5 & 403.2 \\
\hline 25.00 & 454.3 & 458.9 & 461.1 & 460.5 & 456.8 & 449.5 & 438.2 & 422.2 & 400.6 \\
\hline 30.00 & 455.5 & 460.1 & 462.2 & 461.4 & 457.4 & 449.7 & 437.7 & 420.7 & 397.8 \\
\hline 35.00 & 456.7 & 461.3 & 463.2 & 462.3 & 458.0 & 449.8 & 437.1 & 419.2 & 395.0 \\
\hline 40.00 & 457.8 & 462.4 & 464.3 & 463.1 & 458.5 & 449.8 & 436.5 & 417.7 & 392.2 \\
\hline 45.00 & 458.9 & 463.4 & 465.2 & 463.9 & 458.9 & 449.8 & 435.8 & 416.0 & 389.2 \\
\hline 50.00 & 460.0 & 464.5 & 466.2 & 464.6 & 459.4 & 449.8 & 435.1 & 414.4 & 386.2 \\
\hline 55.00 & 461.1 & 465.5 & 467.1 & 465.3 & 459.8 & 449.7 & 434.3 & 412.6 & 383.1 \\
\hline \multirow[t]{2}{*}{60.00} & 462.1 & 466.5 & 468.0 & 466.0 & 460.1 & 449.6 & 433.5 & 410.8 & 380.0 \\
\hline & & & & & MS & & & & \\
\hline 0.10 & 450.3 & 453.8 & 455.9 & 456.4 & 455.2 & 452.5 & 448.1 & 442.1 & 434.5 \\
\hline 1.00 & 450.4 & 454.0 & 456.0 & 456.5 & 455.4 & 452.6 & 448.3 & 442.3 & 434.6 \\
\hline 5.00 & 450.8 & 454.4 & 456.6 & 457.1 & 456.0 & 453.3 & 449.0 & 443.0 & 435.3 \\
\hline 10.00 & 451.2 & 455.0 & 457.2 & 457.8 & 456.8 & 454.2 & 449.8 & 443.8 & 436.1 \\
\hline 15.00 & 451.6 & 455.5 & 457.8 & 458.5 & 457.6 & 454.9 & 450.6 & 444.6 & 436.8 \\
\hline 20.00 & 452.0 & 456.0 & 458.4 & 459.2 & 458.3 & 455.7 & 451.3 & 445.3 & 437.4 \\
\hline 25.00 & 452.4 & 456.4 & 458.9 & 459.8 & 458.9 & 456.3 & 452.0 & 445.9 & 438.0 \\
\hline 30.00 & 452.7 & 456.9 & 459.4 & 460.3 & 459.5 & 457.0 & 452.6 & 446.5 & 438.5 \\
\hline 35.00 & 453.0 & 457.3 & 459.9 & 460.9 & 460.1 & 457.5 & 453.2 & 447.0 & 439.0 \\
\hline 40.00 & 453.3 & 457.6 & 460.3 & 461.3 & 460.6 & 458.1 & 453.7 & 447.5 & 439.4 \\
\hline 45.00 & 453.5 & 458.0 & 460.7 & 461.8 & 461.1 & 458.6 & 454.2 & 447.9 & 439.7 \\
\hline 50.00 & 453.8 & 458.3 & 461.1 & 462.2 & 461.6 & 459.0 & 454.6 & 448.3 & 440.0 \\
\hline 55.00 & 454.0 & 458.6 & 461.5 & 462.6 & 462.0 & 459.4 & 455.0 & 448.6 & 440.2 \\
\hline 60.00 & 454.2 & 458.8 & 461.8 & 463.0 & 462.4 & 459.8 & 455.3 & 448.9 & 440.4 \\
\hline
\end{tabular}




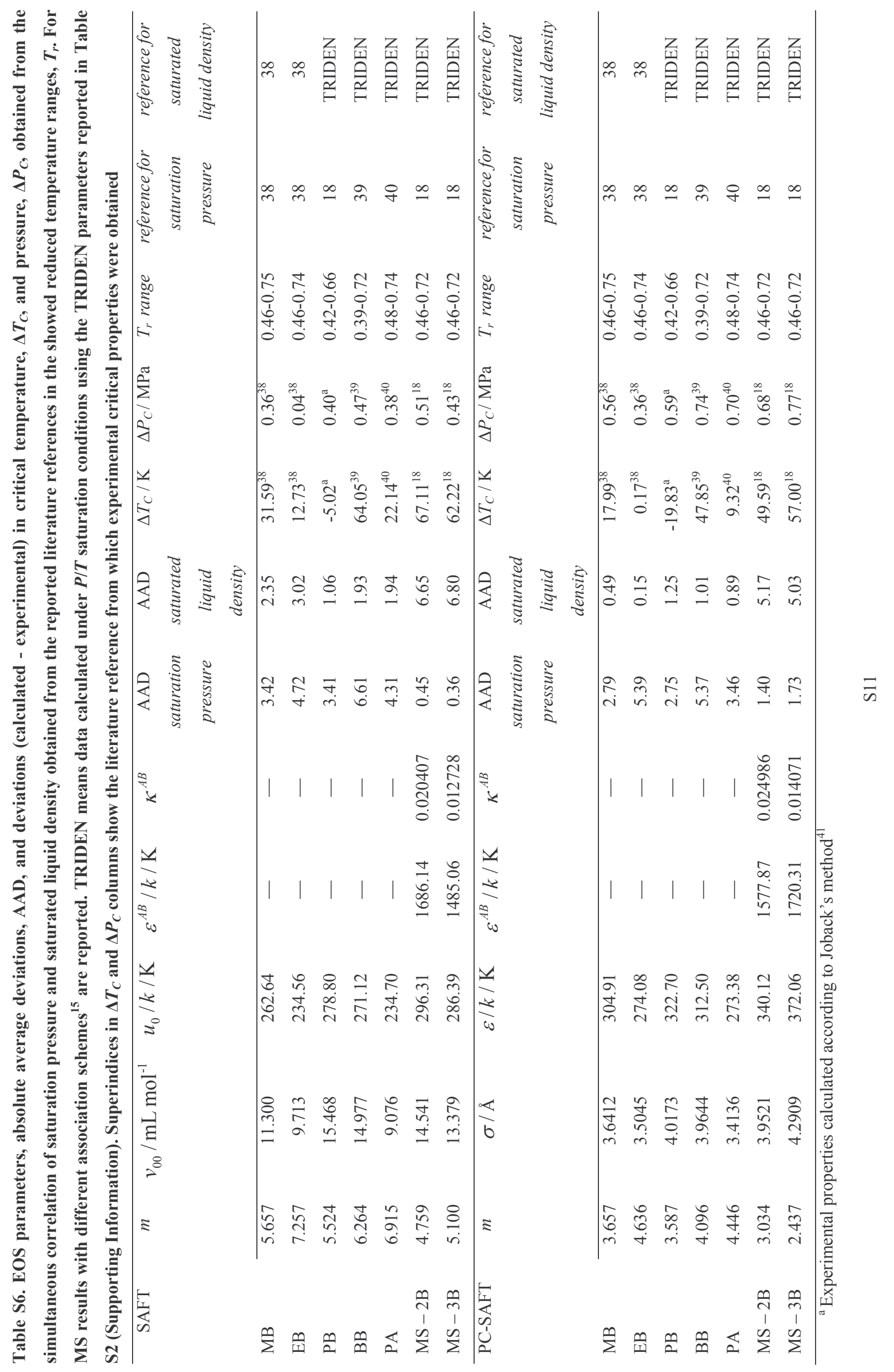


Table S7. Aromatic esters parameters used in the IEF-PCM calculations. $\varepsilon=$ dielectric constant, $\varepsilon_{\infty}\left(=n_{\mathrm{D}}^{2}\right)$ dielectric constant at infinite frequency, $R=$ solvent radius and $d=$ solvent particle density.

\begin{tabular}{lcccccc}
\hline & MB & EB & PB & BB & PA & MS \\
\cline { 2 - 7 }$\varepsilon^{\mathrm{a}}$ & 6.6 & 6.0 & 5.8 & 5.5 & 2.4 & 8.8 \\
$\varepsilon_{\infty}^{\mathrm{b}}$ & 2.29 & 2.26 & 2.25 & 2.24 & 2.25 & 2.36 \\
$R / \AA^{\mathrm{c}}$ & 5.93 & 6.21 & 6.45 & 6.66 & 5.95 & 5.98 \\
$d / \AA^{-3 \mathrm{c}}$ & 0.00478963 & 0.00417463 & 0.00372668 & 0.00338101 & 0.00473611 & 0.00465729
\end{tabular}

${ }^{\mathrm{a}}$ Ref. 56; ${ }^{\mathrm{b}}$ From refractive indices reported in Ref. 11a, except MS for which $n_{D}$ was obtained from Ref. 56; ${ }^{\mathrm{c}}$ From density data at $298.15 \mathrm{~K}$ and $0.1 \mathrm{MPa}$ reported in Table S1. 